\title{
Modulation of the Readily Releasable Pool of Transmitter and of Excitation-Secretion Coupling by Activity and by Serotonin at Aplysia Sensorimotor Synapses in Culture
}

\author{
Yali Zhao and Marc Klein \\ Clinical Research Institute of Montreal and University of Montreal, Montreal, Quebec, Canada H2W IR7, and Department \\ of Physiological Science, University of California Los Angeles, Los Angeles, California 90095-1606
}

\begin{abstract}
Short-term homosynaptic depression and heterosynaptic facilitation of transmitter release from mechanoreceptor sensory neurons of Aplysia are involved in habituation and sensitization, respectively, of defensive withdrawal reflexes. We investigated whether synaptic transmission is regulated in these forms of plasticity by means of changes in the size of the pool of transmitter available for immediate release [the readily releasable pool (RRP)] or in the efficacy of release from an unchanging pool. Using sensorimotor synapses formed in cell culture, we estimated the number of transmitter quanta in the RRP from the asynchronous release of neurotransmitter caused by application of a hypertonic bathing solution. Our experiments indicate that the transmitter released by action potentials and by hypertonic solution comes from the same pool. The RRP was reduced after homosynaptic depression of the EPSP by low-frequency stimulation and increased after facilitation of the EPSP by application of the endog-
\end{abstract}

enous facilitatory transmitter serotonin (5-HT) after homosynaptic depression. However, although the fractional changes in the RRP and in the EPSP were similar for both synaptic depression and facilitation when depression was induced by repeated hypertonic stimulation, the changes in the EPSP were significantly greater than the changes in the RRP when depression was induced by repeated electrical stimulation. These observations indicate that homosynaptic depression and restoration of depressed transmission by $5-\mathrm{HT}$ are caused by changes in both the amount of transmitter available for immediate release and in processes involved in the coupling of the action potential to transmitter release.

Key words: Aplysia; serotonin; habituation; dishabituation; sensitization; transmitter release; synapses; multivesicular release; synaptic ultrastructure; synaptic plasticity; synaptic vesicles; releasable pool; excitation-secretion coupling; homosynaptic depression; synaptic facilitation
Homosynaptic depression and heterosynaptic facilitation at sensorimotor synapses of Aplysia are involved in the modification of defensive withdrawal reflexes by experience (Castellucci et al., 1970; Pinsker et al., 1970, 1973; Carew and Kandel, 1973). Shortterm depression and facilitation are thought to result from modulation of transmitter release from the sensory neurons (Castellucci and Kandel, 1974, 1976; Dale and Kandel, 1990; Armitage and Siegelbaum, 1998). In previous work, based on statistical and kinetic analyses of synaptic transmission, we showed that these forms of synaptic plasticity are attributable to the switching off and on of a subpopulation of release sites rather than by graded changes in release at all sites (Royer et al., 2000). In the present report, we examine the involvement of changes in the readily releasable pool (RRP) of transmitter, which probably represents the population of docked and primed synaptic vesicles (Stevens and Tsujimoto, 1995; Rosenmund and Stevens, 1996; von Gersdorff et al., 1996; Schikorski and Stevens, 1997, 2001), in these forms of synaptic modulation. Alterations in the size of the RRP have been implicated in several forms of plasticity at synapses and in adrenal chromaffin cells (Gillis et al., 1996; Goda and Stevens, 1998; Stevens and Sullivan, 1998; Wang and Kaczmarek, 1998;

\footnotetext{
Received Aug. 22, 2002; revised Oct. 2, 2002; accepted Oct 3, 2002.

This work was supported by Natural Sciences and Engineering Research Council of Canada Grant OGP0138426 and National Institutes of Health Grant NS 36648. We thank Dr. David Glanzman, Dr. Dean Buonamono, Dr. Geoffrey Murphy, and Dr. Felix Schweizer for helpful comments on a previous version of this manuscript. Correspondence should be addressed to Marc Klein, Department of Physiological Science, University of California Los Angeles, 621 Charles E. Young Drive South, Los Angeles, CA 90095-1606. E-mail: kleinm@physci.ucla.edu.

Copyright (C) 2002 Society for Neuroscience 0270-6474/02/2210671-09\$15.00/0
}

Wang and Zucker, 1998; Waters and Smith, 2000). At the same time, we determine whether modulation is limited to transmitter release evoked by electrical stimulation and calcium influx or whether other steps in the release process are also affected.

Previous ultrastructural (Bailey and Chen, 1988) and modeling (Gingrich and Byrne, 1985) studies suggested that depletion of synaptic vesicles contributes to homosynaptic depression at the sensorimotor synapses. On the other hand, Eliot et al. (1994b) concluded, on the basis of their observations of spontaneous transmitter release, that depletion was unlikely to contribute to homosynaptic depression. Here we describe experiments that bear on the question of depletion as a mechanism of homosynaptic depression in the context of modulation of the RRP in synaptic plasticity.

To examine changes in the RRP that accompany homosynaptic depression and restoration of depressed transmission by the facilitatory transmitter serotonin (5-HT), we measured the amount of transmitter released in response to hypertonic stimuli after depression and facilitation of the EPSP. Our results indicate that both the pool of transmitter available for release and processes involved in excitation-secretion coupling are modulated in shortterm synaptic plasticity induced by electrical activity and by a modulatory transmitter.

\section{MATERIALS AND METHODS}

Preparation of cultures. Adult Aplysia californica (75-150 gm; Marine Specimens Unlimited, Pacific Palisades, CA; and Alacrity Marine Biological Services, Redondo Beach, CA) were anesthetized by injection of $50-100 \mathrm{ml}$ of $385 \mathrm{~mm}$ (isotonic) $\mathrm{MgCl}_{2}$. Tail sensory neurons (Walters et al., 1983) and siphon motor neurons (LFS neurons; Frost and Kandel, 
1995) were isolated and maintained as described previously (Klein, 1994; Coulson and Klein, 1997). Sensory and motor neurons were maintained in separate plastic Petri dishes (Falcon 1008; Becton-Dickinson, Mountain View, CA) at room temperature $\left(21-24^{\circ} \mathrm{C}\right)$ in $10 \%$ Aplysia hemolymph in Leibovitz L15 culture medium (Invitrogen, Grand Island, NY) supplemented with salts (Schacher and Proshansky, 1983). Under these conditions, the neurons retract their processes and become spherical in shape after 1-3 d. A single sensory neuron was then manipulated into contact with each motor neuron, and the pairs were left to incubate at least $1 \mathrm{~d}$, by which time the EPSP amplitude has reached a plateau (Coulson and Klein, 1997). For recording, individual pairs were transferred to a separate Petri dish containing artificial seawater (ASW), where they adhered to the bottom without any special treatment.

Electrophysiology. An Axoclamp 2A amplifier (Axon Instruments) and borosilicate glass micropipettes (tip resistance, 10-20 M 2 ) filled with 2 $\mathrm{M}$ potassium acetate, $\mathrm{pH} 7.5$, were used for intracellular recordings from the LFS neuron. Recordings were performed in ASW (in mM: $460 \mathrm{NaCl}$, $10 \mathrm{KCl}, 11 \mathrm{CaCl}_{2}, 55 \mathrm{MgCl}_{2}$, and $10 \mathrm{HEPES}, \mathrm{pH}$ 7.5). Neuron type was confirmed by the response to release of hyperpolarizing current (Eliot et al., 1994a; M. Klein, unpublished data). Throughout the experiments, the LFS motor neuron was hyperpolarized to $-80 \mathrm{mV}$ in current-clamp mode. To elicit an EPSP in the motor neuron, an extracellular pipette filled with ASW was put into contact with the sensory neuron membrane, and a 2 msec current pulse from a Master 8 stimulator (AMPI, Jerusalem, Israel) was applied.

The hypertonic solution used to elicit asynchronous release consisted of ASW with sucrose added to the desired final concentration. For most experiments, $1 \mathrm{~m}$ sucrose was added to the ASW. The hypertonic solution was applied either with a 5-10 sec puff from a microperfusion system (Warner Instruments) or by direct addition of $50 \mu \mathrm{l}$ of the solution from a hand-held Pipetman onto the neurons. The protocol used to examine EPSPs and asynchronous release consisted of a single EPSP elicited by electrical stimulation of the sensory neuron followed within 1 min by application of the hypertonic solution.

Miniature synaptic potentials (minis) elicited by the hypertonic sucrose were identified visually. As a check on the identification of the minis, we compared our counts with the number of minis detected with a program included in the Axograph software package (Axon Instruments) that uses a sliding template to identify minis (Clements and Bekkers, 1997). We constructed a template from the average of $15-30$ of the clearest minis and used this template to examine the baseline before and after the burst of minis triggered by the hypertonic solution to determine the threshold for mini detection. Threshold was taken as 2.5-3 times the SD of the noise. Minis detected by the program were examined by eye, and obvious false-positive results were excluded. There was no difference between the number of miniature potentials detected by the program and the number identified visually (41 experiments; $p=1.0$; paired $t$ test). We used the counts based on visual detection in preference to the automated method for these and all other experiments, because the computer program was thrown off by baseline shifts that resulted in erroneous identification of minis, which then had to be excluded by visual inspection.

To estimate the number of minis that fell below our detection threshold, we fit the amplitude distribution of the minis with an equation based on a normal distribution of synaptic vesicle diameters that has been used to describe mini distributions in other preparations (Bekkers et al., 1990). This equation adequately fit our data also ( $p>0.05$ using the $\chi^{2}$ test; in most cases, $p>0.9$; see Figs. 1, 2, 6, 7). We found the parameters that gave the best fit (by minimizing the sum of the squared differences) to the largest minis in the first peak in the observed distribution (where the likelihood of correct identification was the greatest) and then compared the number of minis of small amplitude predicted from the extrapolated theoretical distribution with the number we actually detected. On average, we estimate that we missed $\sim 8 \%$ of the minis, depending on the average mini amplitude, but the proportion of minis not counted was the same for each portion of a single experiment (see Fig. 7A).

In most experiments, we observed minis that were much greater than the average amplitude for a given experiment (see Fig. 2). When we detected breaks on the rising limb of these "giant minis," we counted them as two or more events. To take into account the possibility that single-event giant minis represented the release of more than one vesicle, we analyzed these events in two ways in each experiment. First, we counted each event as representing a single quantum and compared the number of events in the control and the experimental conditions. Second, we also treated each giant mini as a multiquantal release event by dividing its amplitude by the average mini amplitude as determined by fitting the first peak in the amplitude distribution with the theoretical curve of Bekkers et al. (1990). As long as the giant minis were treated consistently in any given experiment, fractional changes in release caused by the experimental manipulations were not affected by counting giant minis as single or multiple quanta.

All minis detected during the first $55 \mathrm{sec}$ after application of the hypertonic solution were counted (see Fig. 1C). All applications of hypertonic solution within an experiment were done in the same way, i.e., with either a puff or an aliquot; the results were not affected by the manner of application of the hypertonic solution. The preparation was washed with ASW for 10 min between tests.

The quantal content of EPSPs was estimated by dividing the EPSP amplitude by the average mini amplitude for EPSPs that were $\leq 10 \mathrm{mV}$ in amplitude. For larger EPSPs, the peak EPSP amplitudes for the whole experiment were plotted against the maximal slope of the EPSPs (corresponding to the peak synaptic current), and a partial correction for nonlinear summation was done as follows. The linear relationship between amplitude and maximal slope for the smaller EPSPs was extrapolated to the larger amplitude EPSPs. The amplitudes of the larger EPSPs were then multiplied by a factor that made them fall on the extrapolated line, and the corrected amplitudes were divided by the average mini amplitude to get an estimate of the quantal content. The idea behind this correction is that the maximal slope of the EPSP is affected less by nonlinear summation than the peak, because the voltage at which the maximal slope occurs is much less depolarized than the EPSP peak. Because the maximal slope is still affected by nonlinear summation, albeit to a lesser extent, the quantal content of larger EPSPs will still be underestimated. This underestimate does not affect any of our conclusions, because it leads to underestimates of homosynaptic depression, 5-HT-induced facilitation, and the fraction of the RRP released by a single action potential; all of these would lead us to understate our conclusions.

Two estimates of the quantal amplitude were used in each experiment, by treating giant minis as either single quanta or multiple quanta, respectively. When each mini was counted as a single quantum regardless of amplitude, the average of all the events was used as the average mini amplitude. When giant minis were treated as multiquantal, the quantal amplitude was estimated from the first peak in the amplitude frequency histogram. Aside from differences in the quantal amplitude and in the total number of quanta in the RRP, the two ways of counting gave equivalent results. In particular, the extent of neither synaptic depression nor facilitation of the EPSP was affected, and the fraction of the RRP released with a single action potential was not changed.

To examine facilitation of the EPSP, $50 \mu \mathrm{l}$ of ASW containing $10 \mu \mathrm{M}$ 5-HT was applied onto the neurons $\sim 30 \mathrm{sec}$ before stimulating the sensory neuron. To examine the effects of 5-HT on the asynchronous response, the same concentration of 5-HT in the hypertonic solution was applied within $1 \mathrm{~min}$ after the EPSP, either with a puff from the microperfusion apparatus or in a $50 \mu \mathrm{l}$ aliquot.

Experiments were recorded on a Power Macintosh G3 computer running Axograph (Axon Instruments), and EPSPs and minis were analyzed using the same program.

Data files were transferred to Microsoft Excel version 7 and GraphPad Prism version 2 for statistical analysis and plotting. All data are expressed as mean $\pm \mathrm{SEM}$, and $t$ test comparisons are two-tailed unless otherwise noted.

\section{RESULTS}

\section{The size of the readily releasable pool of transmitter at Aplysia soma $\rightarrow$ soma synapses in culture}

Evidence has been presented at hippocampal synapses in culture that the initial burst of transmitter release caused by a hypertonic solution is an index of the RRP (Rosenmund and Stevens, 1996). As is the case for hippocampal synapses, application of hypertonic solution to Aplysia synapses results in asynchronous quantal release of neurotransmitter that does not require presynaptic depolarization (Figs. 1, 2). As shown for hippocampal synapses, this release is not affected by removal of extracellular calcium (data not shown). In our conditions, the release was slow enough to allow us to count the number of miniature synaptic potentials in response to this treatment (Figs. $1 A, 2 A$ ). In most of the experiments, we applied a solution to which $1 \mathrm{M}$ sucrose had been 
A

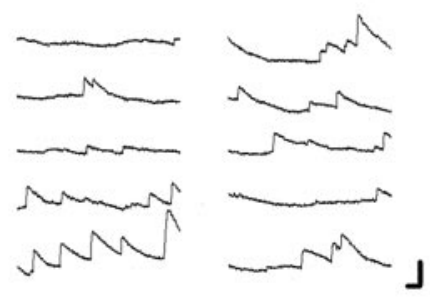

C

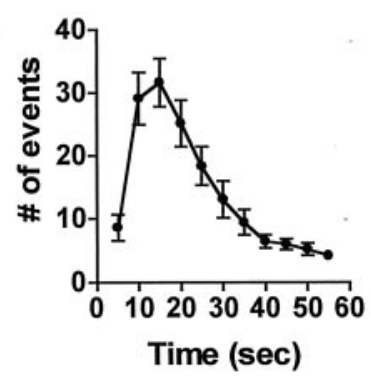

B

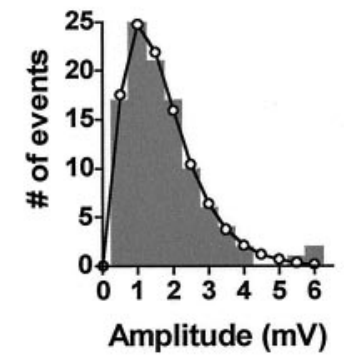

D

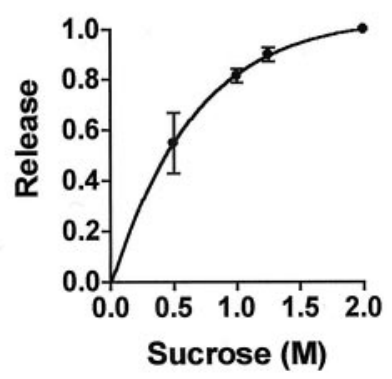

Figure 1. Asynchronous transmitter release triggered by hypertonic solution at Aplysia sensorimotor synapses in culture. $A$, Ten consecutive $1 \mathrm{sec}$ records of miniature synaptic potentials elicited in a postsynaptic neuron $\sim 3$ sec after application of bathing solution with $1 \mathrm{M}$ added sucrose. Calibration: $2 \mathrm{mV}, 100 \mathrm{msec}$. $B$, Amplitude histogram of all the miniature potentials within $1 \mathrm{~min}$ after application of hypertonic solution; same experiment as in $A$. The open circles connected by the line represent the best fit to the experimental points (mean amplitude, $1.67 \mathrm{mV}$ ) using the equation of Bekkers et al. (1990). C, Kinetics of asynchronous transmitter release elicited with hypertonic solution containing $1 \mathrm{M}$ sucrose. Error bars represent SEM for 20 experiments. $D$, Dependence of the asynchronous response on the concentration of added sucrose $(n=6 ; \pm \mathrm{SEM})$. The total number of minis detected in the first $55 \mathrm{sec}$ after application of solutions containing different concentrations of sucrose is plotted against sucrose concentration and normalized to the response at $2 \mathrm{M}$.

added and extrapolated to an estimated maximal response based on the dose-response relationship shown in Figure $1 D$.

In 20 pairs of cells, the estimated maximal number of minis that could be released with hypertonic solution (counted in the first minute after application) (Fig. 1C) ranged from $\sim 60$ to $\sim 640$ (mean, $270 \pm 37$ ). If each mini represents a single quantum of the transmitter, then this is the maximal number of quanta releasable by the hypertonic treatment.

However, the possibility that larger minis represent multiquantal release would increase the estimated size of the RRP, as measured by application of hypertonic solution. As noted in Materials and Methods, most applications of hypertonic solution caused release of minis with amplitudes much greater than the mean. As seen in Figures 2, 6, and 7, these giant minis often appeared to be multiples of the smallest minis, as would be expected if the larger events were multiquantal. Because these large events occurred even when the mean mini frequency was low (Fig. 2A), they do not appear to be fortuitous coincidences of independent release events. If each large mini is taken to represent the release of several quanta, then the total number of quanta releasable by the hypertonic stimulus becomes $571 \pm 128$.
A
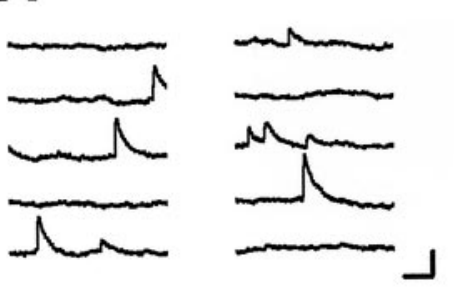

B

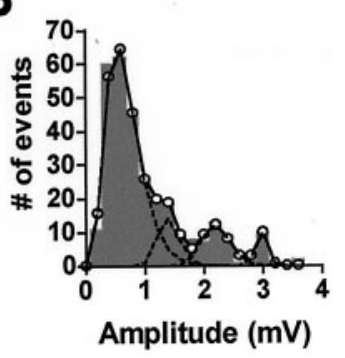

Figure 2. Apparent multiquantal miniature synaptic potentials in response to application of hypertonic solution. $A$, Consecutive $1 \mathrm{sec}$ records from a postsynaptic neuron taken $\sim 40$ sec after application of solution containing $1 \mathrm{M}$ sucrose. The relatively low frequency of minis at this time indicates that the large events are not likely to be coincidences of release from different sites. Calibration: $2 \mathrm{mV}, 200 \mathrm{msec}$. B, Amplitude histogram of all the events recorded within 1 min of sucrose application in the same experiment. Peaks appear at approximately evenly spaced intervals. Circles connected by the solid line represent the fit to the data assuming four peaks with means of $0.67,1.40,2.21$, and $2.97 \mathrm{mV}$, respectively. Broken lines represent fits of the individual peaks. See Figures 6 and 7 for other examples of amplitude histograms with multiple peaks.

In contrast to the persisting steady-state response of the mammalian synapses to the continued presence of hypertonic solution (Stevens and Tsujimoto, 1995; Rosenmund and Stevens, 1996), the asynchronous release evoked in this manner at Aplysia sensorimotor synapses does not continue after the first burst of release (Fig. 1C). The self-limiting nature of the release to hypertonic solution at Aplysia synapses suggests that slow replenishment of the RRP might place a strict limit on the maximal rate of transmitter release even with low rates of stimulation. Because these synapses show marked homosynaptic depression of release at interstimulus intervals as great as 5-10 min (Royer et al., 2000), we examined whether homosynaptic depression is accompanied by a decrease in the size of a slowly recovering releasable pool of transmitter. In addition, because homosynaptic depression can be reversed by application of the endogenous facilitatory transmitter 5-HT, we tested the possibility that this synaptic restoration is accompanied by a corresponding restoration of the releasable pool.

\section{The same pool of transmitter is used in release evoked by electrical stimulation and by hypertonic solution}

To use the release caused by hypertonic solution as a measure of the RRP, it is first necessary to show that the same pool of transmitter is drawn on by electrical excitation and by treatment with hypertonic solution. We therefore attempted to deplete the RRP using high-frequency electrical stimulation and compared the size of the pool determined in this manner with the RRP estimated from application of hypertonic solution to the same synapses. EPSPs evoked at $25 \mathrm{~Hz}$ declined to a low steady-state amplitude by the fourth action potential (Fig. $3 A$ ), suggesting that the RRP had been depleted by the first 3 action potentials and that the EPSPs from this point onward represented a balance between the rate of release and the rate of replenishment of transmitter.

The number of quanta released by a previous test hypertonic stimulus and by the first three action potentials in the train were well correlated (Fig. 3B), but the three action potentials caused release of only $\sim 35 \%$ of the amount of transmitter released by the sucrose solution. The good correlation between the two measures 

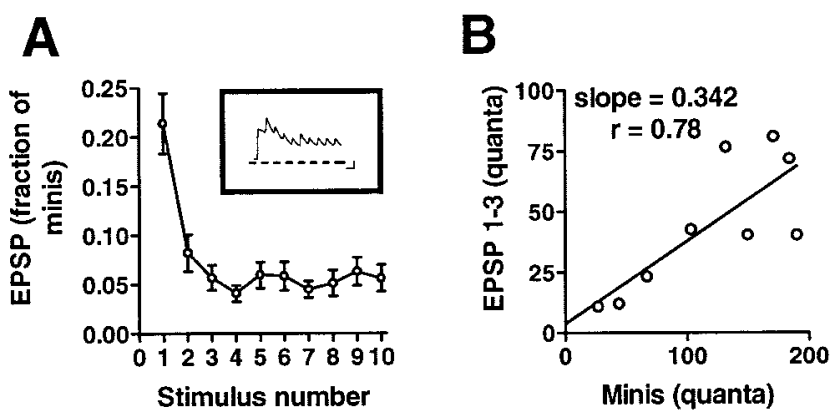

Figure 3. Transmitter release elicited with high-frequency electrical stimulation and with hypertonic solution. A $25 \mathrm{~Hz}$ train of action potentials was triggered with the aim of depleting the RRP, and the RRP was then estimated from the total number of quanta released before the EPSP reached a steady state. $A$, The number of quanta (see Materials and Methods) released in response to each action potential of the train (see inset for sample record; calibration: $5 \mathrm{mV}, 50 \mathrm{msec}$ ) was divided by the total number of quanta released in response to an earlier application of 1 M hypertonic solution $(n=7)$. $B$, The total number of quanta released in response to the first three action potentials of each train is plotted against the total number of quanta released in response to the hypertonic solution $(n=9)$. Although the first three action potentials released only approximately one-third of the amount released with the hypertonic solution, the correlation between the number of quanta released by the action potentials and by the hypertonic stimulus is statistically significant (one-tailed $p<0.01)$.

supports the idea that hypertonic solution and electrical stimulation cause release of transmitter from a common pool, but the quantitative mismatch ( $p=0.0009$; paired Student's $t$ test) suggests that the release caused by action potentials differs in some way from the release triggered by hypertonic solution. There are three possible explanations for this difference. First is the possibility that the two ways of evoking release draw on pools that overlap but are not identical, as would be the case if some vesicles could be released by a hypertonic stimulus but not by an action potential. Second, the pools might be the same, but the apparent larger size of the pool as measured by the hypertonic stimulus might result from refilling of the pool during the longer-lasting hypertonic stimulus. Refilling of the pool during the 1 min measurement period is not likely, because these synapses show no significant recovery from depression for $>10 \mathrm{~min}$ after the stimulation of even one action potential (Royer et al., 2000). Finally, it could be that electrical stimulation leads to a more profound depression of release from the same pool because of progressive uncoupling of the action potential from transmitter release before depletion is reached.

To distinguish among these possibilities, we evoked EPSPs immediately after the asynchronous release caused by $1 \mathrm{M}$ hypertonic sucrose had declined completely. If the pools of transmitter used in the asynchronous response and in the EPSP were the same, depleting the pool with a hypertonic stimulus should severely reduce the subsequent EPSP. On average, the EPSP after the cessation of the asynchronous release was reduced by $\sim 85 \%$ compared with a test EPSP measured immediately before the sucrose application (Fig. 4, Late; $n=8$ ). This reduction compares favorably with the estimated release of $\sim 80 \%$ of the RRP in $1 \mathrm{M}$ sucrose-containing solution (Fig. 1D). In contrast, an EPSP that followed the test EPSP by a comparable interval without an intervening application of sucrose was $80.5 \pm 2.8 \%$ of the test (Fig. 4, Control; $n=8$ ). The large depression of the EPSP by the release caused by hypertonic solution implies that the pool of transmitter used in electrical stimulation is included in the pool

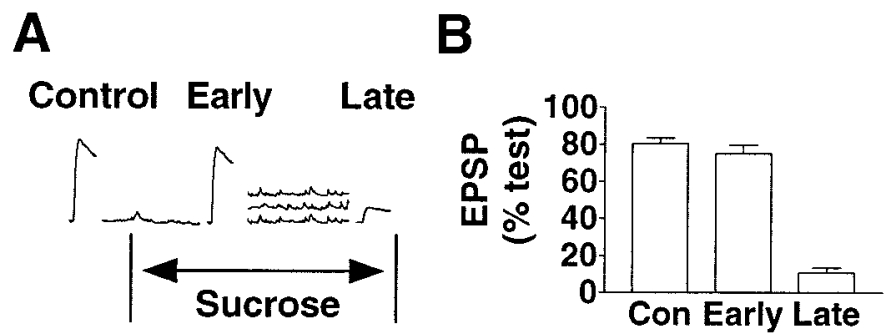

Figure 4. Transmitter release by hypertonic solution reduces the release evoked with an action potential. $A$, Illustration of the experimental protocol. After a single test EPSP was elicited (data not shown), a second EPSP was evoked in one of the following three conditions: (1) without any intervening treatment (Control), (2) in the presence of the hypertonic solution before asynchronous transmitter release had occurred (Early); or (3) in the presence of hypertonic solution immediately after the decay of the barrage of miniature synaptic potentials (Late). B, Summary of experimental results. The EPSP was reduced only if it was triggered after the asynchronous response to the hypertonic stimulus. (Control, $n=8$; Early, $n=12$; Late, $n=8$ ).

drawn on by the hypertonic stimulus, and the quantitative agreement between the reduction in the EPSP and the proportion of the RRP released by the hypertonic stimulus suggest that the two pools might be the same.

An additional experiment tested whether the reduction of the EPSP after the asynchronous response was a consequence of the transmitter release caused by the hypertonic solution or was simply an effect of the hypertonic solution. Because the asynchronous release is delayed by a few seconds (Fig. $1 A, C$ ), we were able to measure EPSPs evoked in the presence of hypertonic solution but before any significant release occurred. The amplitude of EPSPs measured under these circumstances was $74.8 \pm 4.7 \%$ $(n=12)$ of the amplitude of the test EPSPs evoked before the application of the hypertonic solution (Fig. 4, Early, not different from Control; $p=0.759$, Welch's alternate $t$ test, two-tailed), indicating that in the absence of the asynchronous transmitter release, the hypertonic solution itself does not reduce the EPSP.

To test further the idea that the RRP as defined by a hypertonic stimulus is the same as the RRP drawn on by electrical stimulation, we also examined how the response to hypertonic solution was affected by a preceding EPSP. We counted the number of minis in an asynchronous response to a hypertonic stimulus when an EPSP had first been evoked immediately after the application of the hypertonic solution but before the onset of the asynchronous minis. We then compared this number with the number of quanta released by a test hypertonic stimulus presented $10 \mathrm{~min}$ after $(n=6)$ (Fig. $5 A)$ or $10 \mathrm{~min}$ before $(n=3)$ the combined electrical and hypertonic stimulus. There was a significant reduction to $67 \pm 5.0 \%$ of the expected number of quanta in the asynchronous response when it was preceded by an EPSP. Moreover, the sum of the asynchronous quanta and the estimated number of quanta in the EPSP in the experimental situation was close to the number of quanta in the response to the test hypertonic stimulus alone (Fig. $5 B$ ), once again strongly suggesting that a single pool of quanta is drawn on by both kinds of stimulation.

To examine the relationship between the pool of quanta drawn on by electrical stimulation and by the hypertonic stimulus in a slightly different way, we compared the extent of the reduction of the asynchronous response with the amount of release associated with the previous EPSP. If both types of stimulation draw on the same pool, it would be expected that the greater the release in the EPSP, the greater the reduction in the subsequent asynchronous 
A

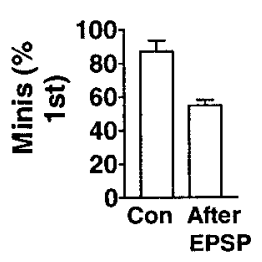

B

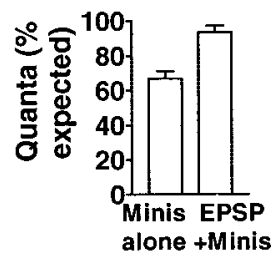

C

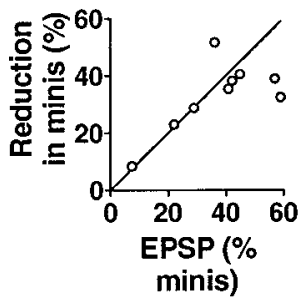

Figure 5. An EPSP reduces the subsequent asynchronous response to hypertonic solution. $A$, A second application of a hypertonic solution 10 min after a first test application gave an asynchronous response that was on average $\sim 87 \%$ of the test response [control (Con); $n=22]$. However, if the second response was immediately preceded by an EPSP, the second response was reduced to $\sim 55 \%$ of the first (After EPSP; $n=6$ ). B, Although the number of quanta in the asynchronous response was reduced by a preceding EPSP (Minis alone), the sum of the number of quanta in the EPSP and in the asynchronous response was equal to the total number expected for a second application of hypertonic solution $(E P S P+$ Minis; $n=9) . C$, In nine experiments, the fractional reduction in the asynchronous response caused by a preceding EPSP was similar to the fraction of the asynchronous response estimated to be released in the EPSP ( $p=0.28$; paired $t$ test), indicating a common pool of quanta for the EPSP and the asynchronous response. The slope of the line is 1.

release. Using the dose-response curve of Figure $1 D$ to estimate the total number of quanta in the RRP releasable by a hypertonic stimulus, we calculated the fraction of this pool that was released in the EPSP and the corresponding reduction in the subsequent asynchronous release. The reduction in the asynchronous release, expressed as a fraction of the estimated RRP, was correlated with the fraction of the pool released by the preceding electrical stimulus $(r=0.685$; one-tailed $p=0.02)$. The fractional reduction in the asynchronous response in each experiment was approximately equal to the fraction of the pool released by the EPSP (Fig. $5 C ; p=0.28$, two-tailed paired Student's $t$ test). Collectively, these results imply that the pool of quanta used in the asynchronous response to hypertonic sucrose is essentially identical to the pool drawn on in the release caused by an action potential.

The conclusion that the same pool of quanta serves in the release to both electrical and hypertonic stimulation implies that the reduction of the EPSP associated with high-frequency stimulation does not result simply from exhaustion of the RRP but is associated with some additional inhibitory process that is triggered by high-frequency electrical stimulation. In the experiment of Figure $3 B$, the total number of quanta released before the EPSP reached a steady state was only approximately one-third of the number of quanta in the response to a hypertonic stimulus. If the same pool is used in both kinds of release, the total number of quanta releasable by action potentials should be similar to the total release caused by the hypertonic solution. Because this prediction is consistently contradicted by the experimental results, we propose that an additional inhibitory process triggered by excitation-secretion coupling is responsible for limiting release after electrical stimulation at high frequency. We now examine whether a similar conclusion holds for the homosynaptic depression that accompanies low rates of electrical stimulation.

\section{Both the readily releasable pool of transmitter and the efficacy of release are decreased in homosynaptic depression}

Having established the legitimacy of using the asynchronous release triggered by a hypertonic stimulus as a measure of the $\mathrm{RRP}$, it is now possible to directly test the hypothesis that this

A

Control
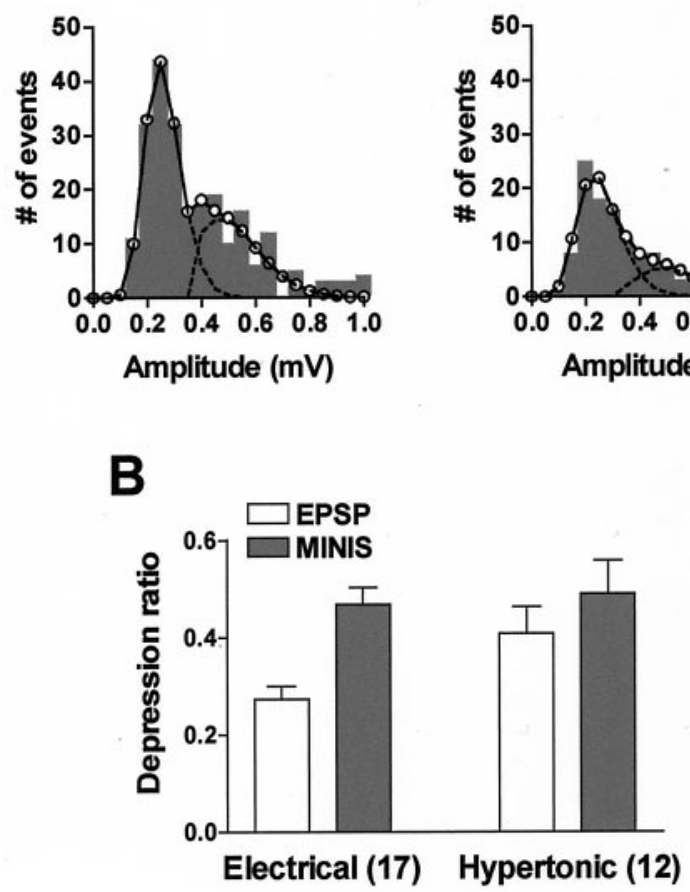

Figure 6. The RRP is reduced in homosynaptic depression. $A$, In the first part of the experiment, a test EPSP was elicited, and the number of minis released in response to a hypertonic stimulus immediately afterward was recorded (Control). After a $10 \mathrm{~min}$ wash and rest, homosynaptic depression was induced by repeatedly eliciting EPSPs at a low frequency. Steady-state homosynaptic depression was tested with an EPSP 2 min after the train, and the response to the hypertonic solution was measured again (Depressed). B, After repeated low-frequency stimulation, the EPSP was reduced more than the number of minis in the asynchronous response relative to the test (Electrical; $0.273 \pm 0.027$ and $0.469 \pm 0.034$, respectively; $p<0.0001$; paired $t$ test). However, when depression was induced by repeated applications of hypertonic solution, the reductions in the EPSP and in the asynchronous response were comparable (Hypertonic; $0.409 \pm 0.055$ and $0.490 \pm 0.068$, respectively; $p>0.2$ ). Numbers in parentheses denote numbers of independent experiments. The depression ratio is the ratio of the EPSP or number of minis in the asynchronous response after depression to their respective test values.

pool is reduced in homosynaptic depression caused by lowfrequency stimulation. If the same pool of transmitter is used in both types of transmitter release, then homosynaptic depression caused by electrical stimulation should reduce the release caused by the hypertonic solution. We found this to be the case. Homosynaptic depression of EPSPs induced by eliciting 15-20 action potentials at a low frequency (interstimulus interval, 10-30 sec) reduced the asynchronous release evoked by hypertonic sucrose (Fig. 6A,B, Electrical), signifying a reduction in the RRP. A corollary of this result is that processes in addition to those triggered by action potentials must be altered in homosynaptic depression.

Transmitter release is normally caused by the calcium influx triggered by an action potential; it is therefore difficult to determine whether transmitter release per se or the calcium influx that causes it is responsible for synaptic depression caused by action potentials. However, the fact that the response to hypertonic stimulation does not require external calcium allows us to ask whether calcium influx is required for homosynaptic depression to occur. We tested this idea by repeatedly applying a hypertonic 
solution and counting the number of quanta released with each application. Repeated hypertonic stimulation resulted in homosynaptic depression of the asynchronous mini response (Fig. $6 B$, Hypertonic). Furthermore, if electrical and hypertonic stimulation cause release from the same pool, then homosynaptic depression caused by repeated hypertonic stimulation should also reduce the transmitter release caused by an action potential. This is what we observed: repeated application of the hypertonic solution reduced the EPSP caused by an action potential as long as 10 min after washout (Fig. 6B, Hypertonic).

There was a difference between the depression induced by action potentials and by repeated sucrose applications, however. When depression was induced by hypertonic stimulation, the declines in the EPSP and in the asynchronous response were quantitatively similar $(p>0.2)$ (Fig. 6B, Hypertonic). In contrast, when depression was induced by action potentials, the decrease in the EPSP was consistently greater than the decrease in the asynchronous release $(p<0.0001)$ (Fig. 6B, Electrical $)$. Furthermore, although the depression of the EPSP by repeated hypertonic stimulation and the depression of the RRP by either type of stimulation were quantitatively similar $(p>0.25$ for each pair of comparisons), the depression of the EPSP by electrical stimulation was greater than the others $(p=0.041$ compared with hypertonic depression of the EPSP; $p=0.010$ compared with hypertonic depression of the RRP). These observations suggest that, as was the case for high-frequency stimulation, there is a component of homosynaptic depression that depends on excitation-secretion coupling, in addition to the decrease in the RRP. This component of depression requires electrical stimulation for both its induction, because it does not accompany the depression induced by hypertonic stimulation, and its expression, because depression induced by electrical stimulation is greater when measured with EPSPs than with hypertonic stimuli.

\section{Restoration of depressed synapses by $5-\mathrm{HT}$ is accompanied by an increase in the RRP and in the efficacy of release}

Transmitter release at depressed synapses can be restored by the endogenous facilitatory transmitter 5-HT acting primarily through the protein kinase C (PKC) pathway (Braha et al., 1990; Ghirardi et al., 1992; Sugita et al., 1992). We therefore investigated whether 5-HT would also increase the response to application of hypertonic solution, suggesting full or partial restoration of the RRP. We found that 5-HT did increase the triggered asynchronous release after homosynaptic depression induced with repeated action potentials (Fig. $7 A, B$, Electrical) and that, as for homosynaptic depression, the relative change in the EPSP was greater than the change in the response to the hypertonic stimulus $(p<0.02)$. Interestingly, 5-HT did not increase either the EPSP or the asynchronous response above its predepression level, suggesting that the action of 5-HT at depressed synapses is to restore the releasable pool to its initial size rather than to increase it above its resting value. It should be noted, however, that we induced synaptic restoration by 5-HT only after the EPSP had been depressed to between 20 and $30 \%$ of its initial value, at which time facilitation is mediated almost completely by PKC; with less profound homosynaptic depression, when facilitation is mediated by the joint actions of PKC and PKA, 5-HT can increase the EPSP and the RRP above their respective initial values (Ghirardi et al., 1992; Klein, 1993).

In view of the quantitative difference between the synaptic depressions induced by electrical and hypertonic stimulation, we
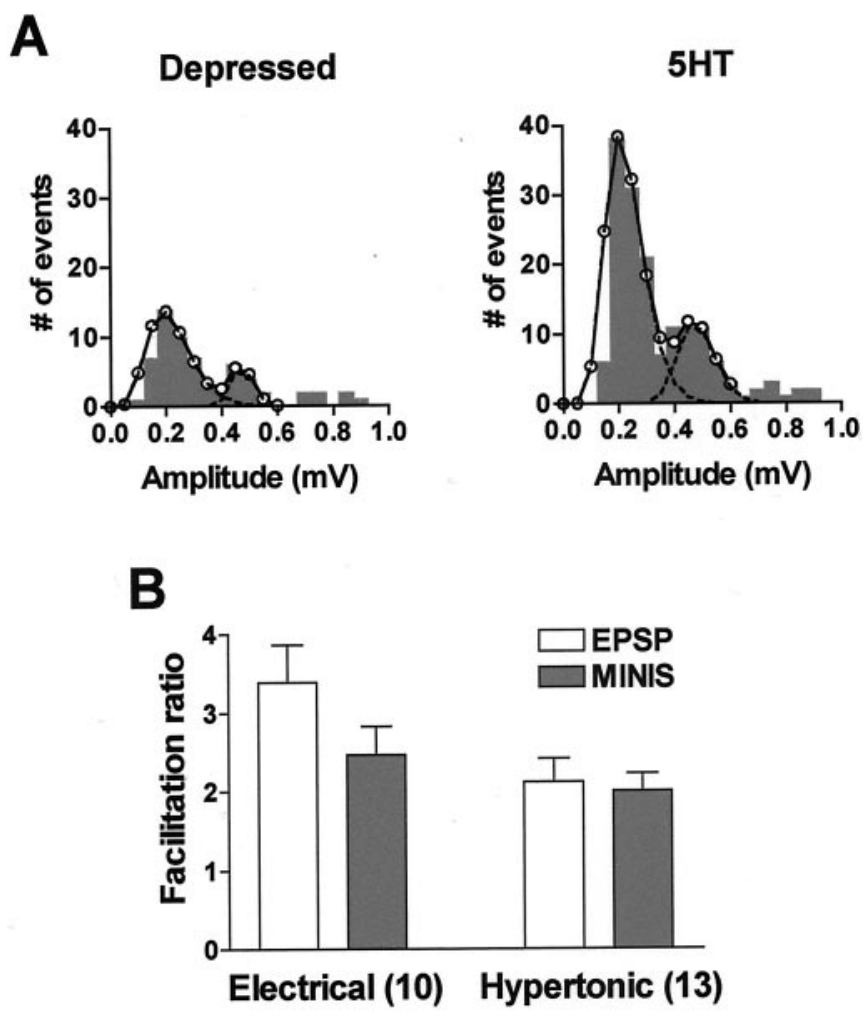

Figure 7. The RRP is increased by 5-HT applied after homosynaptic depression. A, Facilitation of the EPSP by 5-HT after homosynaptic depression induced by electrical stimulation was accompanied by an increase in the response to the hypertonic solution. B, Facilitation of the EPSP was greater than the increase in the RRP after action potentialinduced depression (Electrical; $3.387 \pm 0.474$ and $2.462 \pm 0.368$, respectively; $p<0.02$ ), whereas the facilitation of the EPSP and the increase in the RRP were comparable after depression induced by repeated exposure to the hypertonic solution (Hypertonic; $2.125 \pm 0.287$ and $2.010 \pm 0.213$, respectively; $p>0.6$ ). Facilitation is expressed as the EPSP or number of minis after application of 5-HT relative to the depressed EPSP or number of minis, respectively.

examined whether there was also a difference between the effects of 5-HT when it restored synaptic transmission after the depression caused by each type of stimulation. As we had found for homosynaptic depression, the changes in the EPSP and in the RRP caused by 5-HT after hypertonic depression were quantitatively similar (Fig. 7B, Hypertonic; $p>0.6$ ), in contrast to restoration after electrical depression. Furthermore, the changes in the EPSP after hypertonic depression and in the RRP after both types of depression were similar $(p>0.25$ for all), whereas the facilitation of the EPSP after electrical depression was greater ( $p<0.03$ for all comparisons). These results imply that in the restoration of transmission by 5 -HT also, modulation of both the RRP and excitation-secretion coupling occurs, supporting the idea that this form of facilitation is a reversal of the processes that are responsible for homosynaptic depression (Klein, 1993).

\section{DISCUSSION}

\section{Availability of transmitter and efficacy of release}

We use the terms "availability of transmitter" and "efficacy of release" to refer to the size of the RRP and the fraction of the RRP that is released, respectively. The availability of transmitter is determined by the number of synapses capable of releasing, the number of docking sites at an active zone, and the number of 
releasable vesicles. The efficacy of release is a function of the likelihood that a given synapse will be recruited for release and, at those synapses that are recruited, the probability that a docked and primed vesicle will be released.

Taking the asynchronous release caused by the hypertonic solution as an index of the RRP, we found short-term modulation to be associated with changes both in the availability of transmitter and in the efficacy of release. Changes in the efficacy of release occurred only when depression was induced by action potentials. The finding that changes in the EPSP were consistently greater than the changes in size of the RRP suggests the involvement of an additional process. This idea is supported by our observation that when depression was induced without action potentials, by repeatedly applying hypertonic solution, the changes in the EPSP could be fully accounted for by changes in the pool size.

Although our results were obtained using synapses in culture, they are likely to apply to synapses in intact ganglia. First, unlike mammalian cultures, Aplysia neurons are isolated from adult animals rather than from embryonic tissue, and the synapses formed are thus more likely to resemble adult synapses. Second, comparisons of homosynaptic depression (Eliot et al., 1994b; Royer et al., 2000) and restoration by 5-HT (Royer et al., 2000) between sensorimotor synapses in culture and in situ have shown no significant differences in plasticity between the two preparations. Nevertheless, a definitive analysis of synapses in intact ganglia must await the development of other methods of measuring the RRP.

\section{Spontaneous versus evoked release and transmitter depletion}

Previous studies using cultures investigated the asynchronous release that occurs spontaneously at these synapses in relation to homosynaptic depression and to facilitation by 5-HT (Dale and Kandel, 1990; Ghirardi et al., 1992; Eliot et al., 1994b). Consistent with our results, Dale and Kandel (1990) found that 5-HT increased spontaneous release. In contrast, Eliot et al. (1994b) reported that homosynaptic depression induced by electrical stimulation did not decrease spontaneous release. They therefore suggested that spontaneous and evoked release might not use the same pool of neurotransmitter, as has been suggested for some crayfish and Drosophila synapses (Hua et al., 1998; Koenig and Ikeda, 1999), or else a change in a process specific to release induced by action potentials is responsible for homosynaptic depression. Our results indicate that the RRP is reduced in homosynaptic depression, but our analysis shows that the decrease in the pool is not sufficient to account for the depression induced by electrical stimulation. The failure of Eliot et al. (1994b) to observe a decrease in spontaneous release may have been a consequence of increased intracellular calcium resulting from the high frequency of stimulation $(1 \mathrm{~Hz})$ that they used to induce homosynaptic depression. Although our results agree with those of Eliot et al. (1994b) in suggesting that depletion of the RRP is not sufficient to account for spike-induced homosynaptic depression, our data suggest that depletion of the pool makes a substantial contribution.

Bailey and Chen (1988) found a decrease in the number of docked vesicles in intact ganglia after repeated electrical stimulation, supporting the idea that vesicle depletion is a cause of homosynaptic depression. However, the depletion that they observed is not sufficient to account for homosynaptic depression: When synaptic transmission had been depressed by $>80 \%$, the number of docked vesicles was reduced by only $46-58 \%$. These results are remarkably similar to ours: electrical stimulation caused a depression of $73 \%$ in the EPSP, whereas the response to hypertonic sucrose decreased by only $53 \%$ (Fig. 6B). Approximately one-half of the homosynaptic depression may thus be attributable to a reduced RRP, whereas the remainder is caused by uncoupling of the action potential from release. Refractoriness of the release process, rather than depletion of the vesicle pool, has been suggested to underlie synaptic depression induced by high-frequency stimulation at synapses in vertebrates (Bellingham and Walmsley, 1999; Matveev and Wang, 2000; Waldeck et al., 2000; Brenowitz and Trussell, 2001) and in squid (Hsu et al., 1996), but no detailed models have yet been proposed to account for this refractoriness.

\section{General implications for synaptic transmission}

Our findings suggest that multivesicular release from a single active zone might be the normal mode of transmission at Aplysia sensorimotor synapses, at least for cells in culture. In 20 experiments, the fraction of the RRP that was released with a single action potential was $0.34 \pm 0.04$. If the RRP represents the pool of docked vesicles, then approximately one-third of this pool is released with one action potential. Using complete reconstructions of sensory neuron synapses in abdominal ganglia, Bailey and Chen (1988) counted $\sim 15$ docked vesicles per active zone. Our preliminary estimate of the number of docked vesicles per active zone in soma-to-soma cultures (A. Campbell and Klein, unpublished data) suggests that sensorimotor synapses in culture do not differ fundamentally from those in situ (for electron micrographs of sensorimotor synapses in culture, see Glanzman et al., 1989, their Fig. 5; Klein, 1994, their Fig. 1). A single impulse would then release $\sim 5$ of the 15 docked vesicles at each active zone. If the RRP comprises only a subset of the docked vesicles, then a single action potential would release fewer vesicles, whereas if release occurs at only a subset of synapses, then a single action potential could release more than five vesicles from one active zone.

We favor the conclusion of multiquantal release for the following reasons. First, amplitude histograms of minis, even at low frequencies of release, show peaks that are approximately evenly spaced (Figs. 2, 6, 7). These distributions suggest that multiquantal release events are not uncommon, and it is easier to account for coupled quantal release events occurring at a single active zone than across multiple active zones. Second, if multiquantal release occurs frequently enough, some of these releases could be of a large fraction of the vesicles at one active zone, and the quantal content of the largest minis could then approach the number of docked vesicles. The mean quantal content of the largest amplitude mini in each of 20 experiments was $11.75 \pm 1.90$ (range, 3-40), consistent with the average of 15 docked vesicles seen in the ultrastructural observations. Finally, data from sensorimotor synapses in conventional cultures support our inference that multivesicular release may be the rule. Glanzman et al. (1989) examined the number of boutons in sensorimotor cultures and found that the amplitude of the EPSP was correlated with the number of boutons, averaging $\sim 0.7 \mathrm{mV} /$ bouton. Because the quantal amplitude in these cultures is $\sim 100 \mu \mathrm{V}$ (Dale and Kandel, 1990), each bouton releases approximately seven quanta per action potential, close to the five quanta per action potential that we estimated above. Multivesicular release has now been shown by several groups to occur at various mammalian synapses as well (Tong and Jahr, 1994; Auger et al., 1998; Wadiche and Jahr, 2001; Oertner et al., 2002). 
We have shown previously (Royer et al., 2000) that both homosynaptic depression and 5-HT-induced facilitation of depressed synapses result from all-or-none changes in the binomial parameter $n$, generally taken as the number of release sites (Redman, 1990). This conclusion can be reconciled with our finding of changes in the RRP if $n$, rather than representing the number of active zones (as would be required if release were uniquantal), actually represents the number of docked vesicles.

\section{Possible sites of modulation}

Because 5-HT does not increase resting intracellular calcium (Blumenfeld et al., 1990; Klein, unpublished data), the increase in the RRP caused by 5-HT is not caused by an increase in internal calcium. Candidate mechanisms for the increase in the pool include mobilization of vesicles to release sites (Dale and Kandel, 1990; Klein, 1995), perhaps through regulation of vesicle mobility by the cytoskeleton (Klein, 1995; Angers et al., 2002), or through priming of vesicles that are already docked.

Modulation of the efficacy of release could result from any of several processes. Alteration of calcium influx caused by changes in the calcium conductance or in the shape of the action potential could change excitation-secretion coupling. Another possible target for modulation is the calcium sensor, perhaps synaptotagmin I (Fernandez-Chacon et al., 2001). Finally, action potential firing may regulate the priming of vesicles for calcium-mediated release by controlling the formation of the protein complexes required for exocytosis, for example (Ashery et al., 2000; Betz et al., 2001).

The results presented here, together with our previous findings and work from several other preparations, suggest that all-ornone switching of release sites, multivesicular release, and release-independent synaptic depression play general roles in the modulation of synaptic transmission.

\section{REFERENCES}

Angers A, Fioravante D, Chin J, Cleary LJ, Bean AJ, Byrne JH (2002) Serotonin stimulates phosphorylation of Aplysia synapsin and alters its subcellular distribution in sensory neurons. J Neurosci 22:5412-5422.

Armitage BA, Siegelbaum SA (1998) Presynaptic induction and expression of homosynaptic depression at Aplysia sensorimotor neuron synapses. J Neurosci 18:8770-8779.

Ashery U, Varoqueaux F, Voets T, Betz A, Thakur P, Koch H, Neher E, Brose N, Rettig J (2000) Munc13-1 acts as a priming factor for large dense-core vesicles in bovine chromaffin cells. EMBO J 19:3586-3596.

Auger C, Kondo S, Marty A (1998) Multivesicular release at single functional synaptic sites in cerebellar stellate and basket cells. J Neurosci 18:4532-4547.

Bailey CH, Chen M (1988) Morphological basis of short-term habituation in Aplysia. J Neurosci 8:2452-2459.

Bekkers JM, Richerson GB, Stevens CF (1990) Origin of variability in quantal size in cultured hippocampal neurons and hippocampal slices. Proc Natl Acad Sci USA 87:5359-5362.

Bellingham MC, Walmsley B (1999) A novel presynaptic inhibitory mechanism underlies paired pulse depression at a fast central synapse. Neuron 23:159-170.

Betz A, Thakur P, Junge HJ, Ashery U, Rhee JS, Scheuss V, Rosenmund C, Rettig J, Brose N (2001) Functional interaction of the active zone proteins Munc13-1 and RIM1 in synaptic vesicle priming. Neuron 30:183-196.

Blumenfeld H, Spira ME, Kandel ER, Siegelbaum SA (1990) Facilitatory and inhibitory transmitters modulate calcium influx during action potentials in Aplysia sensory neurons. Neuron 5:487-499.

Braha O, Dale N, Hochner B, Klein M, Abrams TW, Kandel ER (1990) Second messengers involved in the two processes of presynaptic facilitation that contribute to sensitization and dishabituation in Aplysia sensory neurons. Proc Natl Acad Sci USA 87:2040-2044.

Brenowitz S, Trussell LO (2001) Minimizing synaptic depression by control of release probability. J Neurosci 21:1857-1867.

Carew TJ, Kandel ER (1973) Acquisition and retention of long-term habituation in Aplysia: correlation of behavioral and cellular processes. Science 182:1158-1160.

Castellucci VF, Kandel ER (1974) A quantal analysis of the synaptic depression underlying habituation of the gill-withdrawal reflex in Aplysia. Proc Natl Acad Sci USA 71:5004-5008.

Castellucci V, Kandel ER (1976) Presynaptic facilitation as a mechanism for behavioral sensitization in Aplysia. Science 194:1176-1178.

Castellucci V, Pinsker H, Kupfermann I, Kandel ER (1970) Neuronal mechanisms of habituation and dishabituation of the gill-withdrawal reflex in Aplysia. Science 167:1745-1748.

Clements JD, Bekkers JM (1997) Detection of spontaneous synaptic events with an optimally scaled template. Biophys J 73:220-229.

Coulson RL, Klein M (1997) Rapid development of synaptic connections and plasticity between sensory neurons and motor neurons of Aplysia in cell culture: implications for learning and regulation of synaptic strength. J Neurophysiol 77:2316-2327.

Dale N, Kandel ER (1990) Facilitatory and inhibitory transmitters modulate spontaneous transmitter release at cultured Aplysia sensorimotor synapses. J Physiol (Lond) 421:203-222.

Eliot LS, Hawkins RD, Kandel ER, Schacher S (1994a) Pairing-specific, activity-dependent presynaptic facilitation at Aplysia sensory-motor neuron synapses in isolated cell culture. J Neurosci 14:368-383.

Eliot LS, Kandel ER, Hawkins RD (1994b) Modulation of spontaneous transmitter release during depression and post-tetanic potentiation of Aplysia sensory-motor neuron synapses isolated in culture. J Neurosci 14:3280-3292.

Fernandez-Chacon R, Konigstorfer A, Gerber SH, Garcia J, Matos MF, Stevens CF, Brose N, Rizo J, Rosenmund C, Sudhof TC (2001) Synaptotagmin I functions as a calcium regulator of release probability. Nature 410:41-49.

Frost WN, Kandel ER (1995) Structure of the network mediating siphon-elicited siphon withdrawal in Aplysia. J Neurophysiol 73:2413-2427.

Ghirardi M, Braha O, Hochner B, Montarolo PG, Kandel ER, Dale N (1992) Roles of PKA and PKC in facilitation of evoked and spontaneous transmitter release at depressed and nondepressed synapses in Aplysia sensory neurons. Neuron 9:479-489.

Gillis KD, Mossner R, Neher E (1996) Protein kinase C enhances exocytosis from chromaffin cells by increasing the size of the readily releasable pool of secretory granules. Neuron 16:1209-1220.

Gingrich KJ, Byrne JH (1985) Simulation of synaptic depression, posttetanic potentiation, and presynaptic facilitation of synaptic potentials from sensory neurons mediating gill-withdrawal reflex in Aplysia. J Neurophysiol 53:652-669.

Glanzman DL, Kandel ER, Schacher S (1989) Identified target motor neuron regulates neurite outgrowth and synapse formation of Aplysia sensory neurons in vitro. Neuron 3:441-450.

Goda Y, Stevens CF (1998) Readily releasable pool size changes associated with long term depression. Proc Natl Acad Sci USA 95:1283-1288.

Hua SY, Raciborska DA, Trimble WS, Charlton MP (1998) Different VAMP/synaptobrevin complexes for spontaneous and evoked transmitter release at the crayfish neuromuscular junction. J Neurophysiol 80:3233-3246.

Hsu SF, Augustine GJ, Jackson MB (1996) Adaptation of $\mathrm{Ca}^{2+}$. triggered exocytosis in presynaptic terminals. Neuron 17:501-512.

Klein M (1993) Differential cyclic AMP dependence of facilitation at Aplysia sensorimotor synapses as a function of prior stimulation: augmentation versus restoration of transmitter release. $\mathrm{J}$ Neurosci 13:3793-3801.

Klein M (1994) Synaptic augmentation by 5HT at rested Aplysia sensorimotor synapses: independence of action potential prolongation. Neuron 13:159-166.

Klein M (1995) Modulation of ion currents and regulation of transmitter release in short-term synaptic plasticity: the rise and fall of the action potential. Invert Neurosci 1:15-24.

Koenig JH, Ikeda K (1999) Contribution of active zone subpopulation of vesicles to evoked and spontaneous release. J Neurophysiol 81:1495-1505.

Matveev V, Wang XJ (2000) Implications of all-or-none synaptic transmission and short-term depression beyond vesicle depletion: a computational study. J Neurosci 20:1575-1588.

Oertner TG, Sabatini BL, Nimchinsky EA, Svoboda K (2002) Facilitation at single synapses probed with optical quantal analysis. Nat Neurosci 5:657-664.

Pinsker H, Kupfermann I, Castellucci V, Kandel E (1970) Habituation and dishabituation of the gill-withdrawal reflex in Aplysia. Science 167:1740-1742.

Pinsker HM, Hening WA, Carew TJ, Kandel ER (1973) Long-term sensitization of a defensive withdrawal reflex in Aplysia. Science 182:1039-1042.

Redman S (1990) Quantal analysis of synaptic potentials in neurons of the central nervous system. Physiol Rev 70:165-198.

Rosenmund C, Stevens CF (1996) Definition of the readily releasable pool of vesicles at hippocampal synapses. Neuron 16:1197-1207.

Royer S, Coulson RL, Klein M (2000) Switching off and on of synaptic sites at Aplysia sensorimotor synapses. J Neurosci 20:626-638.

Schacher S, Proshansky E (1983) Neurite regeneration by Aplysia neu- 
rons in dissociated cell culture: modulation by Aplysia hemolymph and the presence of the initial axonal segment. J Neurosci 3:2403-2413.

Schikorski T, Stevens CF (1997) Quantitative ultrastructural analysis of hippocampal excitatory synapses. J Neurosci 17:5858-5867.

Schikorski T, Stevens CF (2001) Morphological correlates of functionally defined synaptic vesicle populations. Nat Neurosci 4:391-395.

Stevens CF, Sullivan JM (1998) Regulation of the readily releasable vesicle pool by protein kinase C. Neuron 21:885-893.

Stevens CF, Tsujimoto T (1995) Estimates for the pool size of releasable quanta at a single central synapse and for the time required to refill the pool. Proc Natl Acad Sci USA 92:846-849.

Sugita S, Goldsmith JR, Baxter DA, Byrne JH (1992) Involvement of protein kinase $\mathrm{C}$ in serotonin-induced spike broadening and synaptic facilitation in sensorimotor connections of Aplysia. J Neurophysiol 68:643-651.

Tong G, Jahr CE (1994) Multivesicular release from excitatory synapses of cultured hippocampal neurons. Neuron 12:51-59. von Gersdorff H, Vardi E, Matthews G, Sterling P (1996) Evidence that vesicles on the synaptic ribbon of retinal bipolar neurons can be rapidly released. Neuron 16:1221-1227.

Wadiche JI, Jahr CE (2001) Multivesicular release at climbing fiberPurkinje cell synapses. Neuron 32:301-313.

Waldeck RF, Pereda A, Faber DS (2000) Properties and plasticity of paired-pulse depression at a central synapse. J Neurosci 20:5312-5320.

Walters ET, Byrne JH, Carew TJ, Kandel ER (1983) Mechanoafferent neurons innervating tail of Aplysia. I. Response properties and synaptic connections. J Neurophysiol 50:1522-1542.

Wang C, Zucker RS (1998) Regulation of synaptic vesicle recycling by calcium and serotonin. Neuron 21:155-167.

Wang LY, Kaczmarek LK (1998) High-frequency firing helps replenish the readily releasable pool of synaptic vesicles. Nature 394:384-388.

Waters J, Smith SJ (2000) Phorbol esters potentiate evoked and spontaneous release by different presynaptic mechanisms. J Neurosci 20:78637870 . 